\title{
Aggravated Postinfarct Heart Failure in Type 2 Diabetes Is Associated with Impaired Mitophagy and Exaggerated Inflammasome Activation
}

\author{
Thota Durga Devi, * Mohan Babu, ${ }^{*}$ Petri Mäkinen, * Minna U. Kaikkonen, ${ }^{*}$ Merja Heinaniemi, ${ }^{*}$ Hanne Laakso, * \\ Elias Ylä-Herttuala, ${ }^{*}$ Lassi Rieppo, ${ }^{\dagger}$ Timo Liimatainen, ${ }^{* \ddagger}$ Nikolay Naumenko, ${ }^{*}$ Pasi Tavi, ${ }^{*}$ and Seppo Ylä-Herttuala* ${ }^{\star \delta}$
}

From the Department of Biotechnology and Molecular Medicine, ${ }^{*}$ A.I. Virtanen Institute, the Department of Applied Physics, ${ }^{\dagger}$ the Clinical Imaging Center, ${ }^{\ddagger}$ and the Heart Center and Gene Therapy Unit,$^{\S}$ Kuopio University Hospital, Kuopio, Finland

\author{
Accepted for publication \\ August 24, 2017. \\ Address correspondence to \\ Seppo Ylä-Herttuala, M.D., \\ Ph.D., F.E.S.C., Department of \\ Molecular Medicine, A.I. Vir- \\ tanen Institute, University of \\ Eastern Finland, P.O. Box \\ 1627, FIN-70211 Kuopio, \\ Finland. E-mail: seppo. \\ ylaherttuala@uef.fi.
}

\begin{abstract}
Type 2 diabetes mellitus (T2DM) is a major risk factor for heart disease. Mortality rates after myocardial infarction (MI) are significantly increased in T2DM patients because of dysfunctional left ventricle (LV). However, molecular pathways underlying accelerated heart failure (HF) after MI in T2DM remain unclear. We investigated the underlying mechanisms by inducing MI in a well-established model of T2DM and control mice. Cardiac imaging revealed a significantly decreased global left ventricular ejection fraction in parallel with increased mortality after MI in T2DM mice compared with control mice. Genome-wide mRNA sequencing, immunoblot, electron microscopy, together with immunofluorescence staining for LC3 and p62 indicated an impaired mitophagy in peri-infarct regions of LV in T2DM mice compared with control mice. Furthermore, defective mitophagy was associated with an increased release of mitochondrial DNA, resulting in Aim2 and NLRC4 inflammasome and caspase-I hyperactivation in cardiomyocytes and cardiac macrophages in peri-infarct regions of LV in T2DM mice. Consistent with inflammasome and caspase-I hyperactivation, cardiomyocyte death and IL-18 secretion were increased in T2DM mice. Our results indicate that T2DM aggravates HF after MI through defective mitophagy, associated exaggerated inflammasome activation, cell death, and IL-18 secretion, suggesting that restoring mitophagy and inhibiting inflammasome activation may serve as novel targets for the prevention and treatment of HF in T2DM. (Am J Pathol 2017, 187: 2659-2673; https://doi.org/10.1016/ j.ajpath.2017.08.023)
\end{abstract}

Type 2 diabetes mellitus (T2DM) together with hyperlipidemia and hypercholesterolemia are major risk factors for cardiovascular diseases, including coronary heart disease that contributes to increased rates of morbidity and mortality. $^{1-3}$ Diabetic patients are susceptible to myocardial infarction (MI) due to extensive coronary atherosclerosis. In addition, diabetes substantially accelerates the development of heart failure (HF) and death in patients and animal models with MI. ${ }^{4-6}$ Morbidity, mortality, and re-infarction rates are 2 to 4 times higher after MI in diabetic patients than in nondiabetic subjects, with 1-year mortality rate in this population as high as $50 \% .^{7}$

Despite recent progress in coronary intervention strategies, mortality rates because of MI in diabetic patients are significantly increased because of dysfunctional left ventricle $(\mathrm{LV}) .^{8,9}$ MI leads to increased death of cardiomyocytes and initiates inflammatory responses, contributing to cardiomyocyte dysfunction and subsequently to HF. ${ }^{10}$ At the organ level, LV undergoes a complex remodeling process to compensate for the reduced cardiac function after MI. Failure to compensate will eventually lead to infarct expansion, global ventricular dilation, myocardial hypertrophy, cardiac fibrosis, and

\footnotetext{
Supported by grants from Finnish Academy Center of Excellence program (S.Y.-H.), Biocenter Kuopio (S.Y.-H.), European Research Council Advanced grant (S.Y.-H.), Finnish Foundation for Cardiovascular Research (S.Y.-H.), and Antti and Tyyne Soininen Foundation (M.B.).

T.D.D. and M.B. contributed equally to this work.

Disclosures: None declared.
} 
finally HF. ${ }^{11,12}$ The cellular and molecular pathways contributing to maladaptive ventricle remodeling and HF after MI in diabetic patients are poorly understood, and uncovering the underlying pathophysiological mechanisms may lead to targeted therapies for diabetic patients with MI.

Herein, we have addressed cellular and molecular pathways underlying left ventricular maladaptive remodeling after MI in a well-established mouse model of insulin resistance and T2DM. ${ }^{13}$ These mice demonstrate typical features of human T2DM, including insulin resistance, hyperglycemia, and mild hyperinsulinemia. In addition, these mice show increased atherosclerotic progression and lesion calcification. Previously, with the use of these T2DM mice we have demonstrated an impaired vascular growth in response to hindlimb ischemia as a consequence of altered macrophage phenotype. ${ }^{14}$ With the use of transcriptional profiling and molecular characterization, we found an impaired mitophagy in cardiomyocytes from the peri-infarct zone of LV in T2DM mice. In addition, impaired mitophagy was associated with an increased release of mitochondrial-DNA (mtDNA)-derived damageassociated molecular patterns (DAMPs) intracellularly and extracellularly. Thus, impaired mitophagy and subsequent inflammasome hyperactivation were associated with an increased cardiomyocyte death and proinflammatory cytokine secretion (IL-18) that contributed to maladaptive left ventricular remodeling and HF after MI. Modulation of mitophagy together with inhibition of inflammasome activation may offer a novel therapeutic approach for the prevention of $\mathrm{HF}$ after MI in T2DM.

\section{Materials and Methods}

\section{Mouse Model}

Both male and female mice aged between 12 and 14 months were used in the study (12 to 15 mice per group per time point). All animal experiments were approved by the Experimental Animal Committee, University of Eastern Finland, and procedures were conducted in accordance with approved animal protocols. Mice overexpressing $I G F I I$ in pancreatic beta cells in hyperlipidemic $\left(L D L R^{-/-} A p o B^{100 / 100}\right)$ background $\left(I G F I I / L D L R^{-1-} A p o B^{100 / 100}\right)$ with type 2 diabetic features were fed with high-fat diet for 12 weeks (TD 88173; Boxmeer, NL, 42\% of calories from fat and $0.15 \%$ from cholesterol, no sodium cholate; Harlan Teklad, Madison, WI), ${ }^{13}$ with $C 57 B L / 6 J$ mice fed with regular chow-diet (R36; Lactamin, Stockholm, Sweden) serving as controls.

\section{Mouse MI}

All mice were randomly selected for MI operations. MI was induced by ligation of the left anterior descending coronary artery (LAD) as described previously. ${ }^{15}$ Mice were subjected to small left thoracotomy under general anesthesia with isoflurane $(2 \%)$. The heart was temporarily exteriorized, and a 6.0 silk suture was placed $2 \mathrm{~mm}$ below the origin of the LAD. The heart was replaced immediately into the thoracic cavity, and all thoracic air was evacuated to avoid pneumothorax. Sham operations were performed by passing the suture around the LAD without ligation. After MI, the animals remained supervised until fully conscious. The researcher performing MI operations was blinded for mice groups.

\section{MRI}

Functional cardiac parameters were measured by magnetic resonance imaging (MRI) at baseline, day 7, and day 14 after MI. Mice were anesthetized with $1.5 \%$ to $2 \%$ isoflurane in $70 \% \mathrm{~N}_{2}: 30 \% \mathrm{O}_{2}$ throughout the imaging procedure. A pneumatic pillow placed on the side of the mouse was used for respiratory monitoring and gating the imaging. ECG needles were placed subcutaneously on the mouse front paws to monitor the heart rate and to trigger the imaging. Temperature was maintained at $35^{\circ} \mathrm{C}$ to keep body temperature constant. Images were acquired on a $7 \mathrm{~T}$ horizontal-bore Pharmascan (Bruker Biospin, Ettlingen, Germany) with a 38-mm diameter of surface radiofrequency coil. Short-axis of the heart was determined from echocardiography images on three orthogonal planes (transverse, longitudinal, and sagittal). Axial images perpendicular to the long axis were chosen for cine imaging. An orthogonal long-axis slice and a stack of short-axis slices covering the heart from apex to base were acquired using an ECG and respiratory gating with the following parameters: repetition time of 5.2 milliseconds, echocardiography time of 2 milliseconds, slice thickness of $1 \mathrm{~mm}$, matrix size of $192 \times 192$ (in $2.56 \times 2.56 \mathrm{~cm}^{2}$ field-of-view), and 6 to 8 frames per sequence.

\section{Image Analysis}

Image analysis was performed with Aedes (http://aedes.uef. $f i$, accessed February 12, 2016), on MATLAB (Mathworks Inc., Natwick, CA). Manual segmentation of the endocardium and epicardium of the LV was performed to measure global left ventricular functional parameters [enddiastolic (EDV) and end-systolic (ESV) volume]. Ejection fraction (EF) was calculated using the formula $\mathrm{EF}$ $(\%)=[(\mathrm{EDV}-\mathrm{ESV}) / \mathrm{EDV}] \times 100$.

\section{RNA-Sequencing Library Preparation}

Total RNA was isolated from cardiac tissue proximal and distal to the LAD ligation using RNeasy Fibrous Tissue Mini Kit (Qiagen Finland, Helsinki, Finland) according to the manufacturer's instructions. RNA quantity and quality were assessed with Nanodrop. Poly (A)-RNA was selectively enriched with MicroPoly (A) Purist Kit (Ambion, 
Austin, TX). RNA was treated with TURBO DNase (Ambion), fragmented using RNA Fragmentation Reagents (Ambion), and purified by running through P-30 column (Bio-Rad, Hercules, CA). Fragmented RNA was dephosphorylated with Antarctic phosphatase (New England Biolabs, Ipswich, MA), followed by heat-inactivation. Dephosphorylation reactions were mixed with $2 \times$ Novex Tris/borate/EDTA (TBE)-Urea sample buffer (Invitrogen, San Diego, CA), briefly denatured, and loaded on a Novex denaturing $15 \%$ polyacrylamide TBE-urea gel according to the manufacturer's instructions. Fragments of 50 to 300 nucleotides in length were gel purified as described previously. ${ }^{16}$ Following this original protocol, the poly (A)-tailing and cDNA synthesis was performed the next day. However, for reverse transcription oligos with custom barcodes (underlined) were used as follows: 5'-phosCA/TG/AC/GTGATCGTCGGACTGTAGAACTCT/idSp/CAAGCAGAA GACGGCATACGATTTTTTTTTTTTTTTTTTTTVN-3'. After cDNA synthesis, exonuclease was used to catalyze the removal of excess oligos. Enzyme was inactivated, and RNA was hydrolyzed by alkaline treatment $(100 \mathrm{mmol} / \mathrm{L} \mathrm{NaOH})$ and heat $(25 \mathrm{mi}-$ nutes, $95^{\circ} \mathrm{C}$ ). The cDNA fragments of approximately 150 to $200 \mathrm{bps}$ were purified on a Novex denaturing $10 \%$ polyacrylamide TBE-urea gel (Invitrogen). The recovered cDNA was circularized, linearized, and amplified for eight cycles. The final product was ran on Novex 10\% TBE gel, gel purified as above and cleaned-up using chromatin immunoprecipitation DNA clean \& Concentrator Kit (Zymo Research Corporation, Irvine, CA). The library was sequenced for 50 cycles on the Illumina (San Diego, CA) HiSeq 2000 according to the manufacturer's instructions.

\section{RNA-Sequencing Data Analysis}

Single-end sequencing reads were quality controlled with the FastQC software version 0.11.5 (http://www. bioinformatics. babraham.ac.uk/projects/fastqc, last accessed April 2, 2016), and subsequently bases with poor quality scores were trimmed (requiring a minimum 97\% of all bases in one read to have a min phred quality score of 10) with the use of the FastX toolkit version 0.0.13 (http://hannonlab. cshl.edu/fastx_toolkit, accessed April 2, 2016). The TopHat software version 2.0.9 (Johns Hopkins University,
Baltimore, MD) was used for read alignment to the mouse genome (version mm9), accepting up to two mismatches. A transcriptome index was built from genes.gtf file available from the UCSC $\mathrm{mm} 9$ iGenomes database (Illumina). The HOMER toolkit version 4.3 (Salk Institute for Biological Studies, La Jolla, CA; http://homer.salk.edu/ homer) was used for visualization and transcript quantification based on UCSC Refseq annotations. Transcripts expressed at a level rpkm $>1$ in at least three samples were used for statistical analysis with the R/ Bioconductor package edgeR. Estimation of dispersion was performed gene-wise using default settings. Differentially expressed transcripts were identified using the glmLRT function. Transcripts with at least 1.5 -fold change in expression level and adjusted $P<0.005$ (BenjaminiHochberg method using $P$ values from moderated $t$-test) were defined significantly regulated.

\section{Functional Analysis}

Differentially expressed genes in infarct and peri-infarct regions of LV of T2DM mice compared with control mice were subjected to Ingenuity pathways analysis program (Qiagen, Redwood City, CA). Differentially expressed genes were grouped into functional categories by combining data available in National Center for Biotechnology Information gene information and gene expression data in public data sets.

\section{Histologic Examination}

After sacrificing the mice, hearts were perfused with phosphate-buffered saline (PBS) and immersion fixed in $4 \%$ paraformaldehyde/15\% sucrose ( $\mathrm{pH} 7.4)$ for 4 hours and then rinsed in $15 \%$ sucrose ( $\mathrm{pH} 7.4)$ overnight. Paraffinembedded sections ( $5 \mu \mathrm{m}$ thick) were cut transversely for immunohistochemistry as mentioned previously. ${ }^{14}$ Capillaries were stained with rat anti-mouse CD31 (catalog number 550274; dilution 1:50; BD Pharmingen, San Jose, CA.). Capillary density was measured in a blinded manner (T.D.D.) from five different fields from infarct, peri-infarct, and remote zones. Avidin-biotin-horseradish peroxidase system with diaminobenzidine (DAB) color substrate (catalog number PK-6100; Vector Laboratories, Burlingame, CA) was used for signal detection. Collagen content was analyzed from picro-Sirius Red-stained sections. The

Table 1 Baseline Metabolic Parameters

\begin{tabular}{llll}
\hline Parameter & Control & T2DM & $P$ value \\
\hline Body weight, g & $29.94 \pm 0.9589$ & $41.34 \pm 2.482$ & $<0.0001$ \\
Heart/body weight ratio & $0.004961 \pm 0.00042$ & $0.004676 \pm 0.00031$ & $\mathrm{NS}$ \\
Plasma insulin, $\mathrm{ng} / \mathrm{mL}$ & $0.9963 \pm 0.1393$ & $4.793 \pm 0.8413$ & $<0.0001$ \\
Blood glucose, $\mathrm{mmol} / \mathrm{L}$ & $7.783 \pm 0.8264$ & $18.28 \pm 2.932$ & 0.0072 \\
\hline
\end{tabular}

Values are means \pm SD.

NS, not significant; T2DM, type 2 diabetes mellitus. 
A
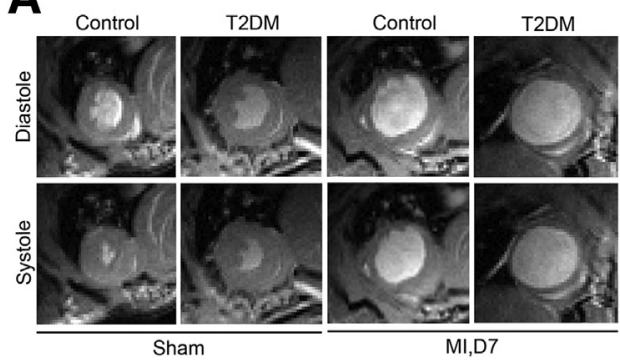

C

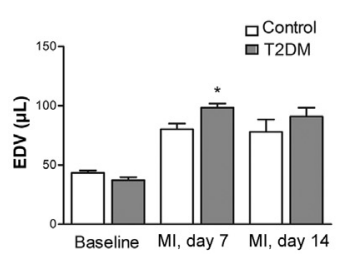

D

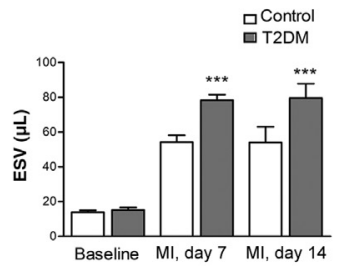

B

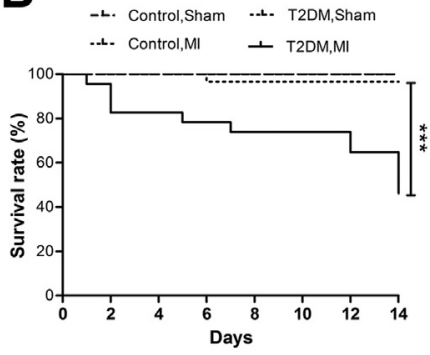

$\mathbf{E}$

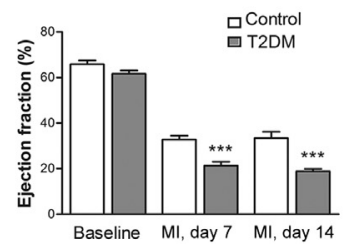

Figure 1 Type 2 diabetes mellitus (T2DM) accelerates heart failure and death after myocardial infarction (MI). A: Representative short-axis magnetic resonance images of sham and infarcted mouse hearts. B: Kaplan-Meier survival curves after MI for control (dotted line) and T2DM (thick line) mice 2 weeks after MI. C-E: Quantification of cardiac parameters, including enddiastolic volume (EDV; C), end-systolic volume (ESV; D), and global ejection fraction (E) at baseline and at day 7 and 14 after MI. Data are expressed as means \pm SEM (C-E). $n=28$ control mice (B); $n=23$ T2DM mice (B); $n=5$ mice $(\mathbf{C}-\mathbf{E})$. Statistical analysis was conducted with one-way analysis of variance with Bonferroni's multiple comparison test. ${ }^{*} P<0.05,{ }^{* *} P<0.005$ T2DM versus controls, log-rank test. percentage of positive stained area was calculated in relation to the whole left ventricular area. Images of stained histologic sections were taken and processed using an Olympus AX 70 microscope (Olympus Optical, Tokyo, Japan) and analySIS version 2.0 (Soft Imaging System, Muenster, Germany) and Photoshop version 11.0.2 (CS4; Adobe, San Jose, CA) softwares. The peri-infarct region within the LV was defined as area containing both infarcted and surviving myocardium within the high power field, whereas the remote region was chosen from a myocardial region without any infarction.

\section{Immunofluorescence Microscopy}

All immunofluorescence stainings were performed on paraffin sections. Macrophages were identified using rat anti-mouse F4/80 antibody (catalog number MCA497; dilution 1:50; AbD Serotec, Oxford, UK). M1-macrophages were detected using $F 4 / 80$ in combination with tumor necrosis factor (TNF)- $\alpha$ antibody (dilution 1:50; catalog number Sc-1351; Santa Cruz Biotechnology, Santa Cruz, CA) and M2-macrophages were detected using F4/80 in combination with arginase I antibody (dilution 1:50; catalog
A

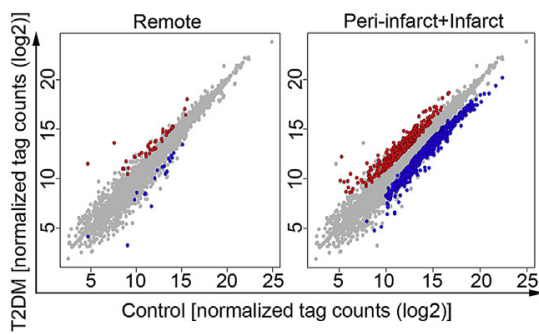

B

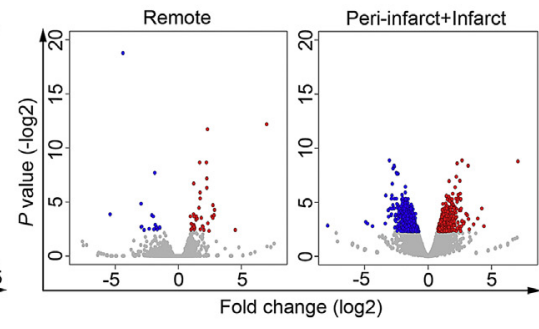

C

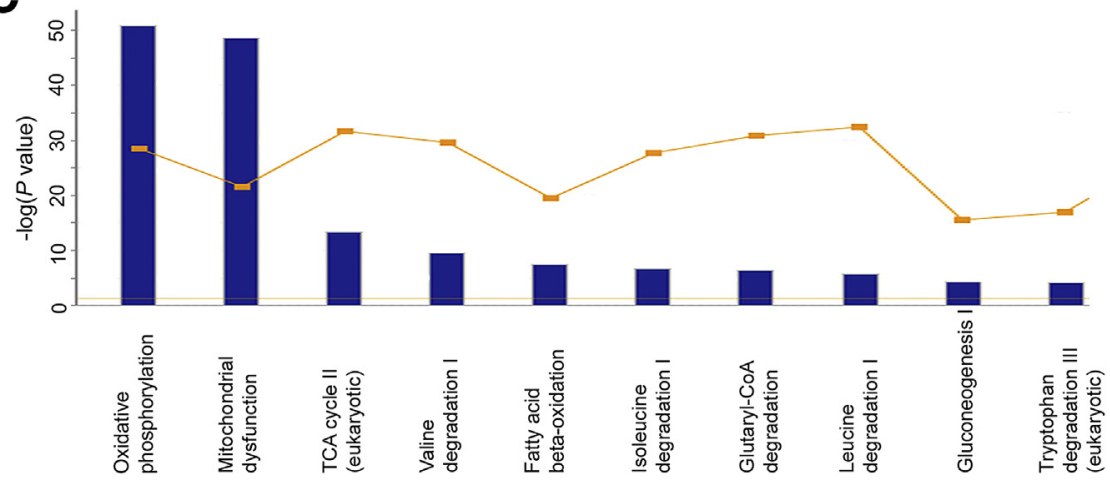

Figure 2 Transcriptomics analysis reveals cellular alterations associated with heart failure (HF) after myocardial infarction (MI) in type 2 diabetes mellitus (T2DM). A and B: Genome-wide mRNA sequencing analysis of left ventricular tissue at day 7 after MI. With the use of a fold change $>1.5$ and an adjusted $P$ value of $<0.005,1609$ genes were found to be differentially expressed (568 up-regulated and 1041 down-regulated) in the peri-infarct and infarct area of left ventricle (LV) of T2DM mice compared with control mice at day 7 after MI as shown in the scatter plots (A) and volcano plots (B). C: Major biological processes altered in peri-infarct and infarct areas from LV of T2DM mice were identified by the Ingenuity pathway analysis. TCA, tricarboxylic acid. 
Table 2 Ingenuity Functional Annotation of Differentially Expressed Genes in Peri-Infarct and Infarcted Left Ventricle of Type 2 Diabetes Mellitus Mice Compared with Controls at Day 7 after Myocardial Infarction

\begin{tabular}{lccc}
\hline Diseases or functions annotation & Z-score & Prediction & Number of genes \\
\hline Cell death & 2.933 & Increased & 344 \\
Necrosis & 2.650 & Increased & 274 \\
Movement disorders & 2.026 & Increased & 136 \\
Dilated cardiomyopathy & 2.113 & Increased & 31 \\
Myocardial dysfunction & 2.107 & Increased & 24 \\
Congestive heart failure & 2.449 & Increased & 16 \\
Muscle contraction & -2.315 & Decreased & 46 \\
Heart rate & -2.419 & Decreased & 55 \\
Metabolism of nucleic acid component & -1.542 & Decreased & 82 \\
\hline
\end{tabular}

MI, myocardial infarction; T2DM, type 2 diabetes mellitus.

number Sc-18354; Santa Cruz Biotechnology). Images of stained histologic sections were taken and processed using an Olympus AX 70 microscope (Olympus Optical).

\section{TUNEL Assay for Apoptosis}

For in situ detection of apoptosis, terminal deoxynucleotidyl transferase-mediated dUTP nick-end labeling (TUNEL) assay
(In Situ Cell Death Detection Kit, Fluorescein, catalog number 1684795; Boehringer, Mannheim, Germany) was used. Mouse myocardial tissue sections were deparaffinized, digested with Proteinase K, and incubated with TdT and fluorescein-labeled dUTP in a humid atmosphere for 60 minutes at $37^{\circ} \mathrm{C}$. After incubation for 30 minutes with an antibody specific for fluorescein-conjugated alkaline phosphatase (Boehringer), the TUNEL stain was visualized with a substrate system in which

Table 3 Transcription Factors and Ligand-Dependent Nuclear Receptors Inhibited and Activated in Peri-Infarct and Infarcted LV of T2DM Mice Compared with Controls at Day 7 after MI

\begin{tabular}{|c|c|c|c|}
\hline Transcription regulator & Activation z-score & $P$ value of overlap & Target genes in data set \\
\hline$\overline{\text { ESRRA }}$ & -2.282 & $5.50^{-17}$ & 29 \\
\hline ESRRG & -2.021 & $1.13^{-4}$ & 9 \\
\hline HAND2 & -2.219 & $1.10^{-1}$ & 5 \\
\hline PPARG & -3.751 & $1.92^{-8}$ & 50 \\
\hline PPARD & -2.807 & $4.91^{-6}$ & 25 \\
\hline PPARA & -2.39 & $8.53^{-7}$ & 46 \\
\hline NR4A3 & -2.299 & $4.61^{-9}$ & 16 \\
\hline TBX5 & -2.377 & $6.96^{-3}$ & 9 \\
\hline PPARGC1A & -5.105 & $4.81^{-18}$ & 47 \\
\hline PPARGC1B & -2.145 & $3.59^{-6}$ & 12 \\
\hline NFE2L2 & -2.531 & $1.26^{-1}$ & 26 \\
\hline SMAD7 & -2.212 & $2.82^{-2}$ & 12 \\
\hline MYC & -2.109 & $1.65^{-5}$ & 83 \\
\hline TRIM24 & -3.148 & $1.54^{-3}$ & 13 \\
\hline NRF1 & -2.433 & $7.27^{-4}$ & 9 \\
\hline WWTR1 & 2 & $1.51^{-2}$ & 4 \\
\hline STAT1 & 2.177 & $1.00^{-0}$ & 13 \\
\hline NR3C2 & 2.588 & $1.53^{-8}$ & 22 \\
\hline SPI1 & 2.62 & $1.87^{-1}$ & 13 \\
\hline NRIP1 & 3.44 & $1.23^{-5}$ & 13 \\
\hline SMAD3 & 2.024 & $6.14^{-2}$ & 17 \\
\hline SERTAD2 & 2.213 & $7.34^{-4}$ & 5 \\
\hline IRF3 & 2.597 & $1.53^{-1}$ & 12 \\
\hline
\end{tabular}

A positive z-score indicates activation, whereas a negative score indicates inhibition.

LV, left ventricle; MI, myocardial infarction; T2DM, type 2 diabetes mellitus. 
A
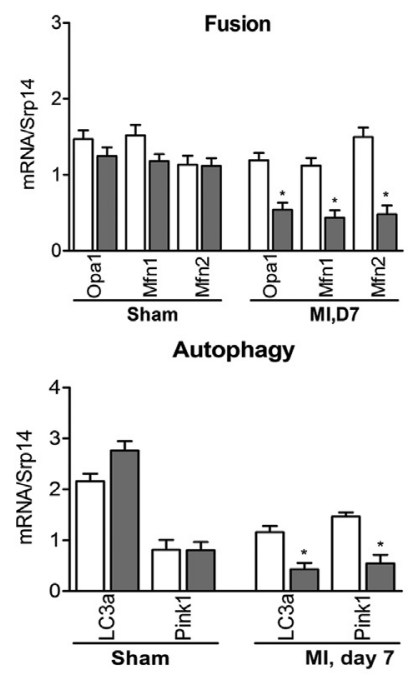

D

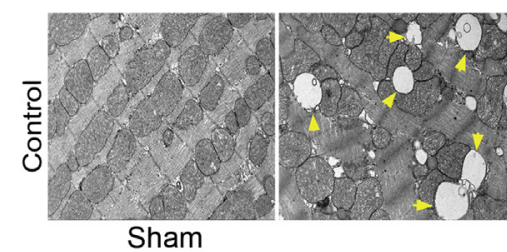

Sham
B
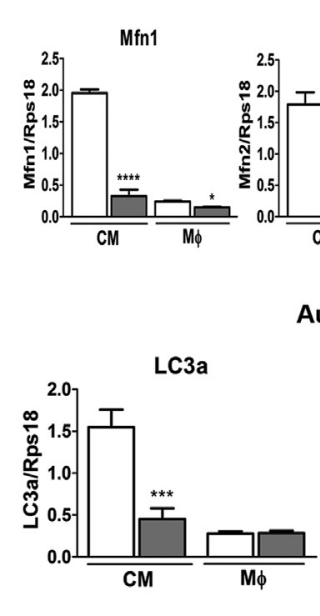

Autophagy

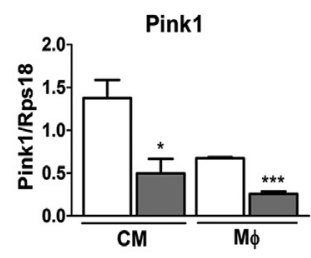

C

Fusion
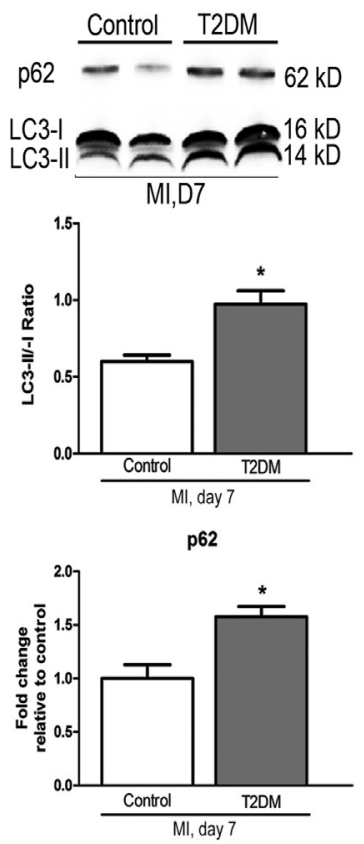

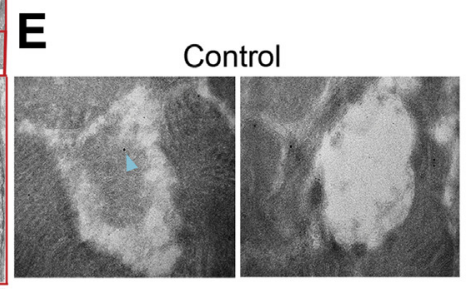

T2DM

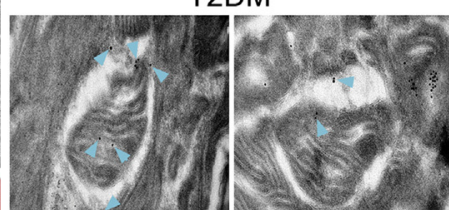

F

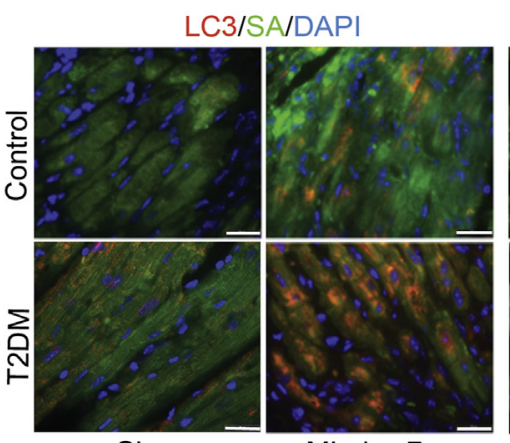

Sham
MI, day 7

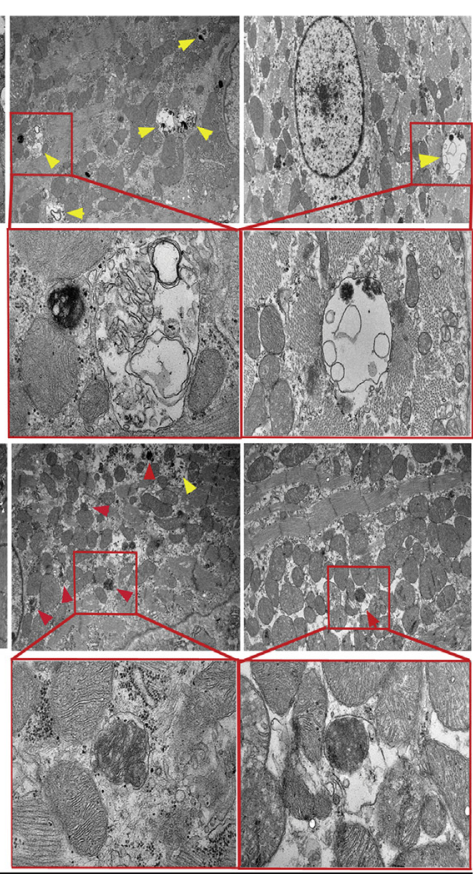

Peri-infarct zone, MI, day 7

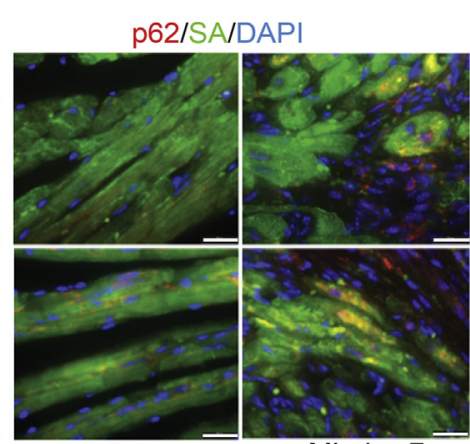

Sham
MI, day 7 
nuclei with DNA fragmentation stain green (Fluorescein substrate system; Dako, Carpinteria, CA). Sections were counterstained with a monoclonal antibody specific for sarcomeric actinin (catalog number A7811; Sigma-Aldrich, Helsinki, Finland). Tissue sections were examined microscopically at $\times 400$ magnification, and TUNEL-positive cells were counted in five high power fields.

\section{Fourier Transform Infrared Spectroscopic Collagen I Mapping}

Collagen I deposition in remodeled cardiac tissues was detected from $5-\mu \mathrm{m}$ thick paraffin sections using Perkin Elmer Spotlight 300 spectrometer (PerkinElmer, Shelton, CT). Data acquisition and analysis were performed as described previously. ${ }^{17}$ The $\mathrm{CH}_{2}$ side chain vibration $\left(1338 \mathrm{~cm}^{-1}\right)$ was used to create collagen I distribution images, and the collagen I area in relation to the whole left ventricular area was determined from these images.

\section{Electron Microscopy}

After scarification and PBS perfusion, left ventricular tissue was quickly cut into 1-mm longitudinal and transverse cubes, immersion fixed in $2.5 \%$ glutaraldehyde in 0.1 $\mathrm{mol} / \mathrm{L}$ phosphate buffer $(\mathrm{pH} 7.4)$ for overnight at $4^{\circ} \mathrm{C}$, and postfixed in $1 \%$ buffered osmium tetroxide. The specimens were dehydrated and embedded in LX-112 resin and polymerized. Toluidine blue staining of semi-thin sections were used to evaluate the orientation of the sections. Ultrathin sections (approximately $70 \mathrm{~nm}$ ) were double stained with lead citrate and uranyl acetate and visualized with transmission electron microscopy (JEM 1200EX; JEOL Ltd., Tokyo, Japan). Autophagosomes or autolysosomes were identified by the characteristic structure of a double or multilamellar smooth membrane completely surrounding compressed mitochondria or membrane-bound electrondense material. ${ }^{18}$

\section{Immunoelectron Microscopy}

Left ventricular tissues were fixed in $4 \%$ paraformaldehyde in $0.1 \mathrm{~mol} / \mathrm{L}$ phosphate buffer, immersed in $2.3 \mathrm{~mol} / \mathrm{L}$ sucrose in PBS, and frozen in liquid nitrogen. Cryo-sections were incubated with mouse monoclonal antibody against double-stranded DNA (dsDNA) (catalog number ab27156; Abcam, Cambridge, UK), followed by incubation with $10 \mathrm{~nm}$ of gold-conjugated secondary antibody (British Biocell International, Cardiff, UK). Control grids were incubated with the secondary antibody alone or with an irrelevant $\operatorname{IgG}$.

\section{Flow Cytometric Analysis of Cardiac Macrophages}

Flow cytometry was performed as described previously. ${ }^{14}$ Infarcted cardiac muscles were minced and enzymatically dissociated using a cocktail that contained $450 \mathrm{U} / \mathrm{mL}$ Collagenase I, $125 \mathrm{U} / \mathrm{mL}$ Collagenase XI, $60 \mathrm{U} / \mathrm{mL}$ DNAseI, and $60 \mathrm{U} / \mathrm{mL}$ hyaluronidase (Sigma-Aldrich) for 1 hour at $37^{\circ} \mathrm{C}$. The cells were then counted and stained for macrophage antigens after initially blocking with rat antimouse CD16/32 mAb (Fc Block; catalog number 553141; BD Pharmingen.) for 10 minutes at $4^{\circ} \mathrm{C}$. To identify M2-like macrophages, we performed staining for $\mathrm{Cd} 45$ (catalog number 103131; BioLegend, San Diego, CA), F4/ 80 (MCA497FB; AbD Serotec), and MRC1 (141707; BioLegend). Fluorescence minus one controls were used to analyze the specific stainings. Fluorescence-activated cell sorting (FACS) was performed on FACSAriaIII (BD Biosciences), and data were analyzed with FCS express 6 (De Novo Software, Glendale, CA).

\section{Adult Cardiomyocyte Isolation}

Mice were sacrificed by rapid neck disarticulation. The thorax was opened, and the aorta was cannulated. Adult mouse ventricular myocytes were obtained by enzymatic dissociation as previously described (AfCS Procedure Protocol PP00000125). ${ }^{19}$ Briefly, isolated hearts were placed in a Langendorff apparatus for perfusion $\left(37^{\circ} \mathrm{C}, 3\right.$ $\mathrm{mL} / \mathrm{min}$ ) with trypsin (Sigma-Aldrich, St. Louis, MO) and liberase (Roche Applied Science, Indianapolis, IN) solution. After perfusion, ventricles were cut into small pieces and gently minced with a Pasteur pipette. The supernatants that contained dissociated cardiomyocytes were filtered through a nylon mesh $(100-\mu \mathrm{m}$ pore size), and the

\footnotetext{
Figure 3 Impaired mitophagy in cardiomyocytes from peri-infarct left ventricle (LV) after myocardial infarction (MI) in type 2 diabetes mellitus (T2DM). A: Expression levels of genes critical in mitochondrial fusion machinery and autophagy in peri-infarct and infarcted left ventricles (LVs) of T2DM mice compared with control mice at day 7 after MI. B: Expression levels of genes critical in mitochondrial fusion and autophagy in cardiomyocytes (CMs) and macrophages isolated from peri-infarct and infarct regions of LV at day 7 after MI. C: Western blot analysis of autophagy proteins LC3 and p62 in myocardial homogenates at day 7 after MI. D: Representative electron microscopic images showing an ordered array of myofibers arranged with mitochondria stacked in between them in CMs of control mice. In addition, intramyocellular lipid deposition can be seen in between mitochondria in the LV of T2DM mice at baseline (black arrows), whereas no lipid droplets were observed in similar regions in control mice. Mitophagy activation showing abundant vacuoles/autolysosomes that contain completely degraded mitochondria (yellow arrowheads) in CMs in peri-infarct regions of LV of control mice, whereas CMs from T2DM mice show an increased accumulation of autophagosomes that contain undegraded mitochondria (red arrowheads). Mitochondria undergoing autophagosomal degradation (red boxed areas) are shown at higher magnification (lower panels). E: Immunoelectron microscopy using anti-DNA antibody showing localization in autolysosomes (blue arrowheads). F: Representative immunofluorescence images showing accumulation of autophagosomes within CMs from peri-infarct areas of LV at day 7 after MI. LC3 and p62 are shown in red and sarcomeric actin in green. Data are expressed as means \pm SEM (A-C). $n=4$ to 5 control mice (A); $n=4$ T2DM mice (A); $n=4$ for each group (B); $n=4$ (C). * $P<0.05$ T2DM versus controls; one-way analysis of variance with Bonferroni's multiple comparison test. Scale bar $=20 \mu \mathrm{m}$. M $\phi$, macrophage; $\mathrm{SA}$, sarcomeric actin.
} 
A

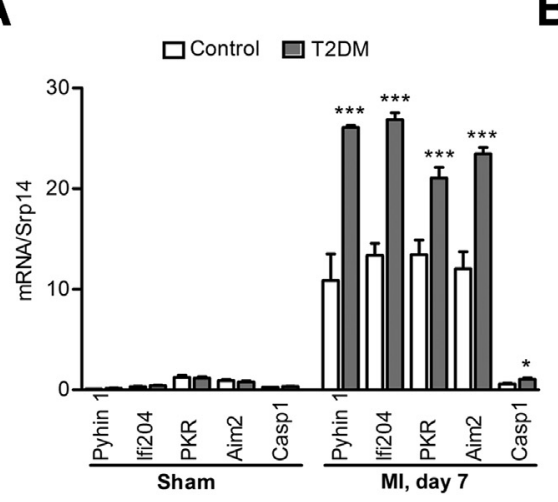

B
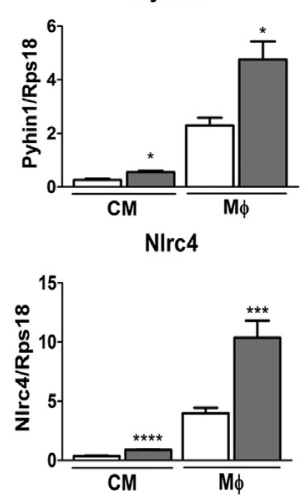

Ifi204
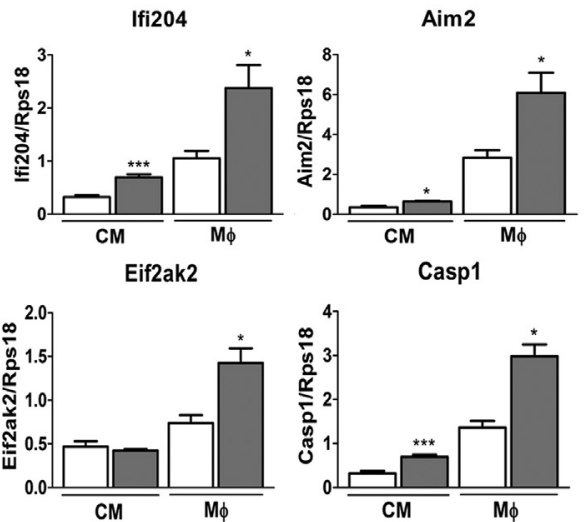

C

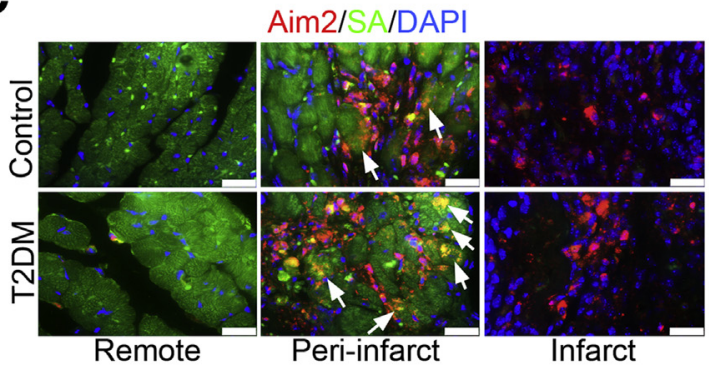

Remote

D

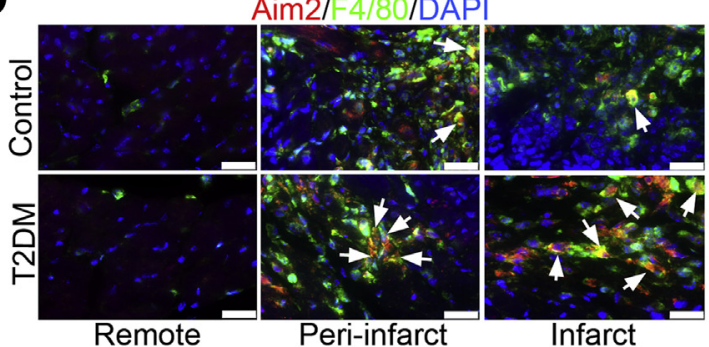

E

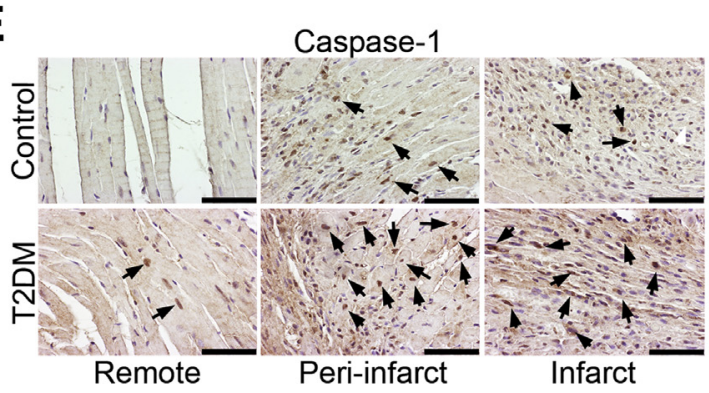

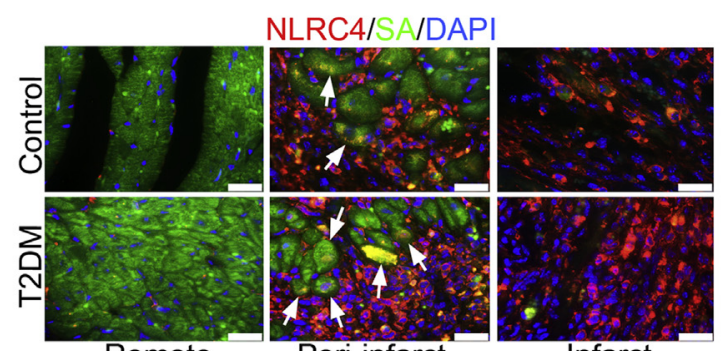

Peri-infarct

Infarct

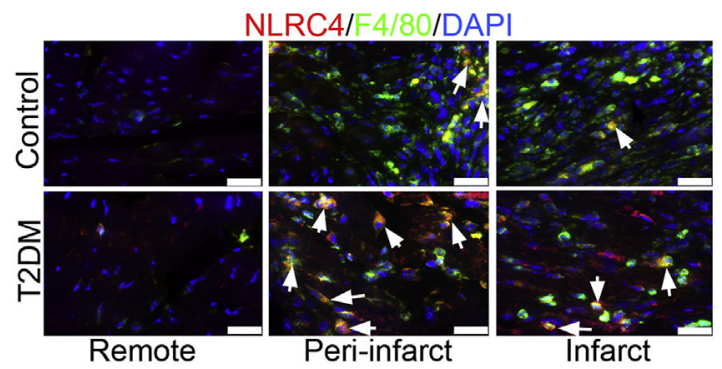

F

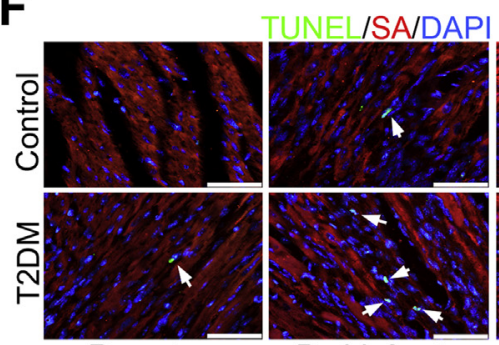

Peri-infarct

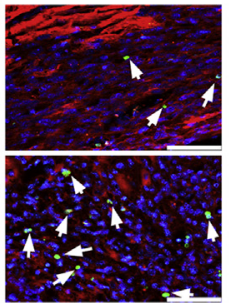

Infarct

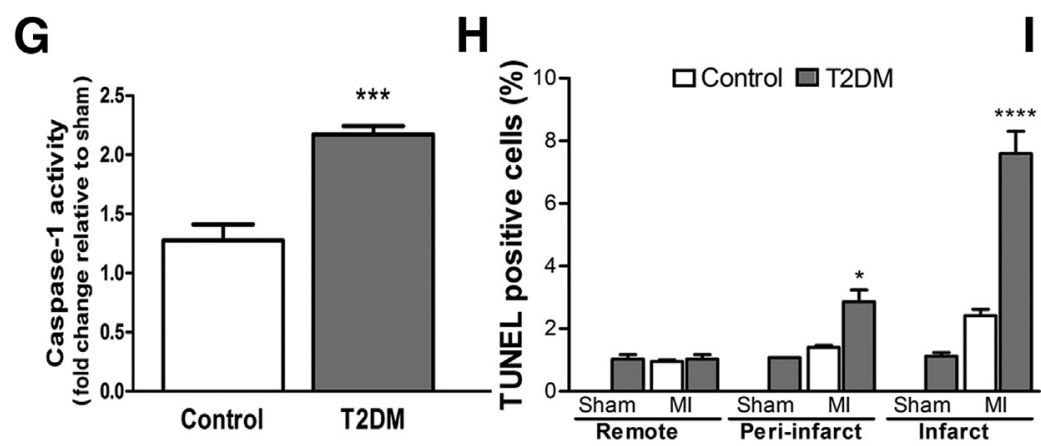

I

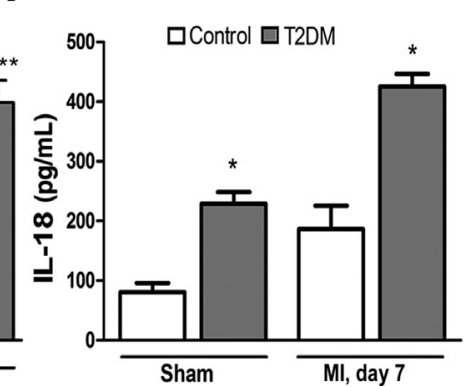


cardiomyocyte fraction was separated by sedimentation by centrifugation at $100 \times g$ for 1 minute.

\section{Flow-Assisted Cell Sorting of Cardiac Macrophages}

Noncardiomyocyte fraction from Langendorff perfusion protocol was subsequently labeled with $\mathrm{CD} 31$ magnetic beads and separated into $\mathrm{CD} 31^{+}$and $\mathrm{CD} 31^{-}$fractions by using CD31 magnetic bead enrichment (catalog number 130-097-418; Miltenyi Biotec, Cologne, Germany). For macrophage sorting $\mathrm{CD} 31^{-}$fraction was incubated for 15 minutes with rat anti-mouse CD16/32 mAb (Fc Block; catalog number 553141; BD Pharmingen) and stained with fluorescein isothiocyanate-conjugated rat antimouse F4/80 antibody (catalog number MCA497F; $\mathrm{AbD}$ Serotec) for 30 minutes at $4^{\circ} \mathrm{C}$. FACS was performed on FACSArialII (BD Biosciences, San Jose, CA) as described previously. ${ }^{14}$

\section{Real-Time Quantitative PCR}

Total RNA isolated from cardiac tissue distal to LAD ligation was reverse transcribed to cDNA with the use of Moloney Murine Leukemia Virus Reverse Transcriptase (Fermentas, Helsinki, Finland). First-strand cDNA was synthesized from $1 \mu \mathrm{g}$ of the extracted RNA in a $20-\mu \mathrm{L}$ reaction volume with the use of random hexamers (Promega Corporation, Madison, WI) as primers. Total RNA was isolated from cardiomyocytes, and macrophages underwent FACS using Arcturus PicoPure RNA isolation Kit (KIT0204; Thermo Fisher Scientific, Waltham, MA). Firststrand cDNA was synthesized from $100 \mathrm{ng}$ of total RNA in a $20-\mu \mathrm{L}$ reaction volume as described above. Gene expression analysis was performed with the Step one plus Real-time PCR system with assays on demand (Applied Biosystems, Foster City, CA).

\section{Western Blot Analysis}

Left ventricular tissue was homogenized in tissue protein extraction reagent supplemented with protease inhibitors and sodium orthovanadate. The homogenate was centrifuged at $12,000 \times g$ for 5 minutes, and the supernatant was collected for downstream analysis. Total protein concentration was measured with the BCA kit (catalog number 23227; Pierce Biotechnology, Rockford, IL). Total protein $(100 \mu \mathrm{g})$ was run on $12 \%$ precast gels (catalog number 4561043; Bio-Rad) and transferred on to polyvinylidene difluoride membrane. The membrane was blocked with $5 \%$ bovine serum albumin in Tris-buffered saline $/ 0.1 \%$ Tween 20 (TBST) for 1 hour at room temperature and then probed with rabbit monoclonal LC3 antibody (catalog number 4599; Cell Signaling, Danvers, MA) and rabbit monoclonal p62 antibody (catalog number TA307334; Origene, Rockville, MD) at $4^{\circ} \mathrm{C}$ overnight. Proteins were detected with horseradish peroxidase-conjugated goat anti-rabbit secondary antibody (catalog number 31460; Thermo Fisher Scientific) in TBST for 1 hour at room temperature, followed by thorough washing with TBST and incubation with enhanced chemiluminescence substrate (catalog number 32106; Pierce Biotechnology).

\section{Statistical Analysis}

Results were expressed as means \pm SEM. Statistical significance was evaluated with one-way analysis of variance, followed by Bonferroni analysis or by $t$-test. All statistical analyses, except RNA-sequencing data analysis, were performed with GraphPad Prism software version 5 (GraphPad Software Inc., La Jolla, CA). A $P<0.05$ was considered statistically significant.

\section{Results}

T2DM Accelerates Heart Failure and Mortality After MI

T2DM mice displayed an increased body weight and plasma insulin and blood glucose levels at baseline. Heart weight-to-body weight ratio was comparable between the groups (Table 1). MI was induced by LAD ligation in control and T2DM mice. In response to MI, a marked increase in the left ventricular chamber dilation was observed in both groups compared with their respective sham controls (Figure 1A). In response to MI, approximately $60 \%$ of all T2DM mice died during the 14 days after MI, whereas only $2.8 \%$ of mice died in the control group during the same period. No deaths were observed in

\footnotetext{
Figure 4 Inflammasome hyperactivation in cardiomyocytes (CMs) and macrophages from peri-infarct and infarct area of left ventricle (LV) after myocardial infarction (MI) in type 2 diabetes mellitus (T2DM). A: Expression profiles of genes involved in cytosolic double-stranded DNA (dsDNA) sensing and inflammasome activation from sham and infarcted LV at day 7 after MI. B: Expression profiles of genes involved in cytosolic dsDNA sensing and inflammasome activation in CMs and macrophages isolated from peri-infarct and infarct areas of LV at day 7 after MI. C and D: Representative images from immunofluorescence double stainings for CM inflammasomes (Aim2, NLRC4) (C) and macrophage inflammasome (Aim2, NLRC4) (D) in LV at day 7 after MI (white arrows). E and G: Representative images from caspase-1 immunostaining of the LV at day 7 after MI (black arrows). In addition, caspase-1 colorimetric assay using left ventricular tissue homogenate is shown. F: Representative apoptotic CMs (green) positive for terminal deoxynucleotidyl transferase-mediated dUTP nick-end

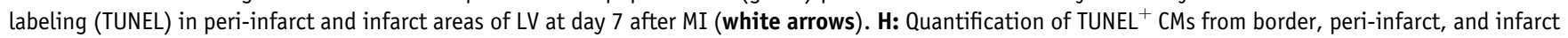
regions of LV from sham and infarcted hearts at day 7 after MI. I: Quantification of caspase-1-dependent inflammatory mediator (IL-18) in circulation at baseline and day 7 after MI. Data are expressed as means \pm SEM (A, B, G-I). $n=5$ control mice (A); $n=4$ T2DM mice $(\mathbf{A}) ; n=4$ for each group $(\mathbf{B}) ; n=4$ to $5(\mathbf{E}-\mathbf{I}) .{ }^{*} P<0.05, * * * P<0.005$, and ${ }^{* * * * P}<0.0001$ T2DM versus controls, one-way analysis of variance with Bonferroni's multiple comparison test. Scale bars: $20 \mu \mathrm{m}$ (C and $\mathbf{D}) ; 50 \mu \mathrm{m}$ (E and $\mathbf{F})$. M $\phi$, macrophage; SA, sarcomeric actin.
} 

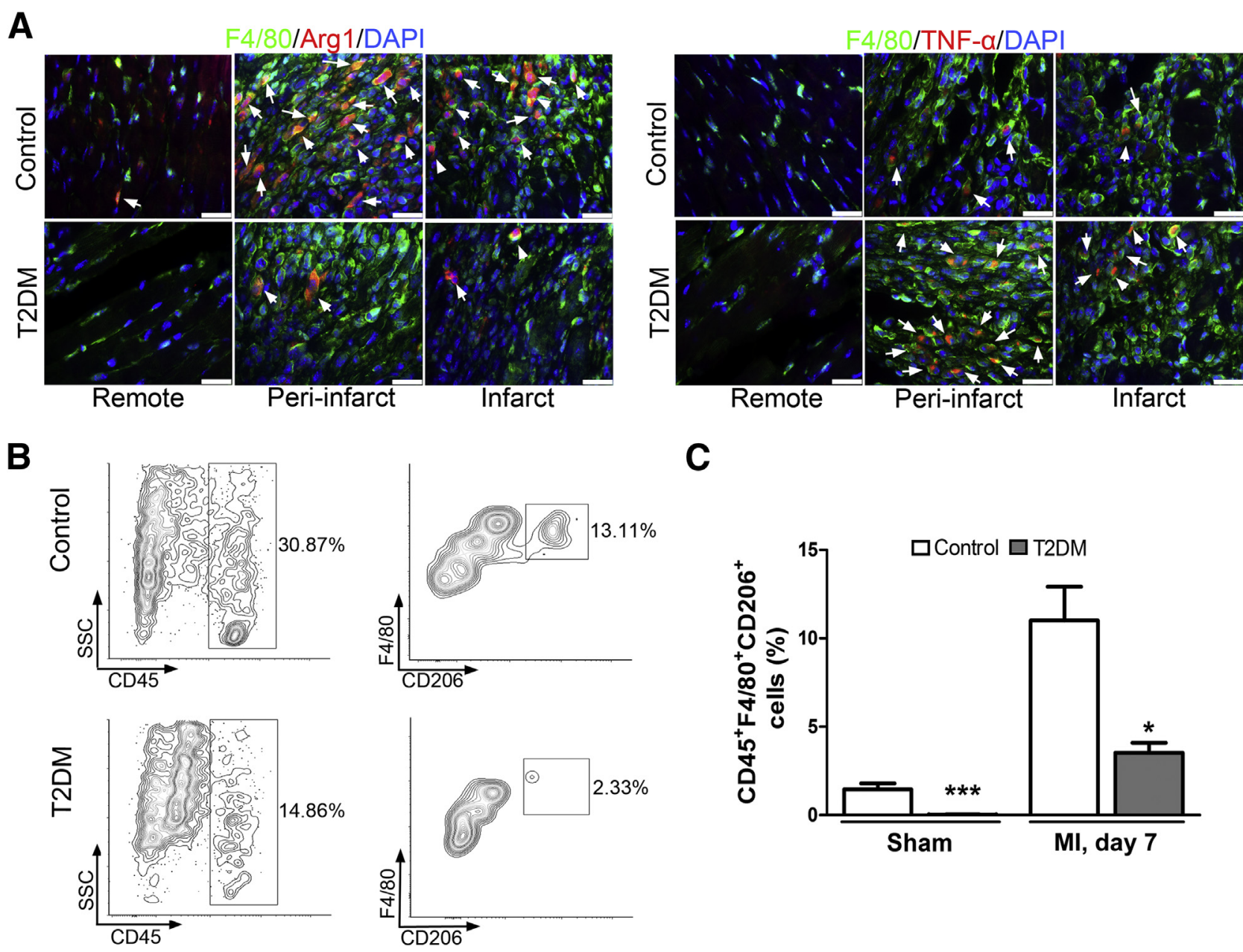

Figure 5 Altered cardiac macrophage phenotype in peri-infarct and infarct area of left ventricle (LV) after myocardial infarction (MI) in type 2 diabetes mellitus (T2DM). A: Double immunofluorescence staining of M2-like macrophages [anti-F4/80 and anti-Arginase-1 (Arg1)] and M1-like macrophages [anti-F4/ 80 and anti-tumor necrosis factor (TNF)- $\alpha$ ] in control and T2DM LVs at day 7 after MI (white arrows). B: Leukocytes were gated with CD45 fluorescence versus side scatter (SSC) area. Scatter plots are gated on $\mathrm{CD} 45^{+}$population. M2-macrophages (CD45 ${ }^{+} \mathrm{F} 4 / 80^{+} \mathrm{CD} 206^{+}$cells) were analyzed from sham and infarcted hearts of control and T2DM mice. C: Quantification of M2-macrophages from left ventricular tissues of sham and infarcted hearts. Data are expressed as means \pm SEM (C). $n=3$ to $4 .{ }^{*} P<0.05,{ }^{* *} P<0.005$ versus controls, one-way analysis of variance with Bonferroni's multiple comparison test. Scale bar $=50 \mu \mathrm{m}$.

the sham-operated groups (Figure 1B). At baseline conditions, T2DM mice showed a mild yet nonsignificant decline in EDV compared with controls, whereas ESVs were similar in both groups. After MI, both groups showed a significant increase in left ventricular EDV and ESV compared with baseline values. T2DM mice displayed a robust increase in EDV and ESV compared with controls after MI (Figure 1, C and D). A significant systolic dysfunction was observed as assessed by left ventricular $\mathrm{EF}$ in mice with MI compared with sham controls $(P<0.005$, MI versus sham). However, systolic dysfunction was exacerbated in T2DM mice compared with controls as exemplified by a significant decline in global left ventricular EF $(P<0.005$ at day 7 and 14 After MI, T2DM MI versus control MI) (Figure 1E). Collectively, these data indicated that T2DM adversely influenced cardiac function and increased mortality rate in response to MI.
RNA-Sequencing Reveals Dysregulation of Genes Related to Cardiac Contractility, Cell Death, and Metabolism of Nucleic Acids in T2DM Hearts

Because T2DM severely impairs tissue repair after injury, and accumulating evidence has suggested alterations in the inflammation resolution phase of tissue repair, genome-wide mRNA sequencing was performed on peri-infarct and infarct regions of left ventricular tissue collected at day 7 after MI. Left ventricular tissue remote to the infarct region served as an internal control. The RNA-sequencing data have been deposited in National Center for Biotechnology Information Gene Expression Omnibus (http://www.ncbi. nlm.nih.gov/geo; accession number GSE69201). By using a threshold of fold change $>1.5$ and an adjusted $P$ value of $<0.005,1609$ genes were found to be differentially expressed in the peri-infarct and infarct regions of LV of T2DM compared with controls. Among them, 568 genes 
A

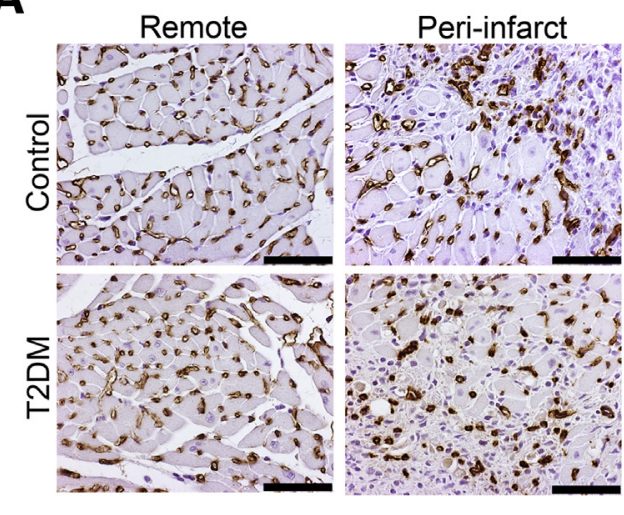

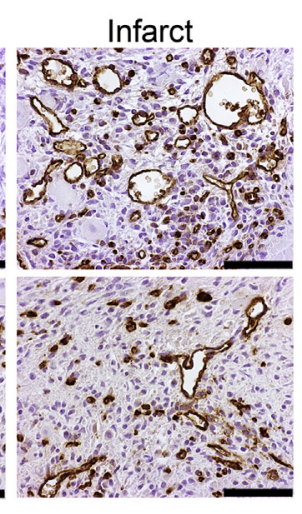

B
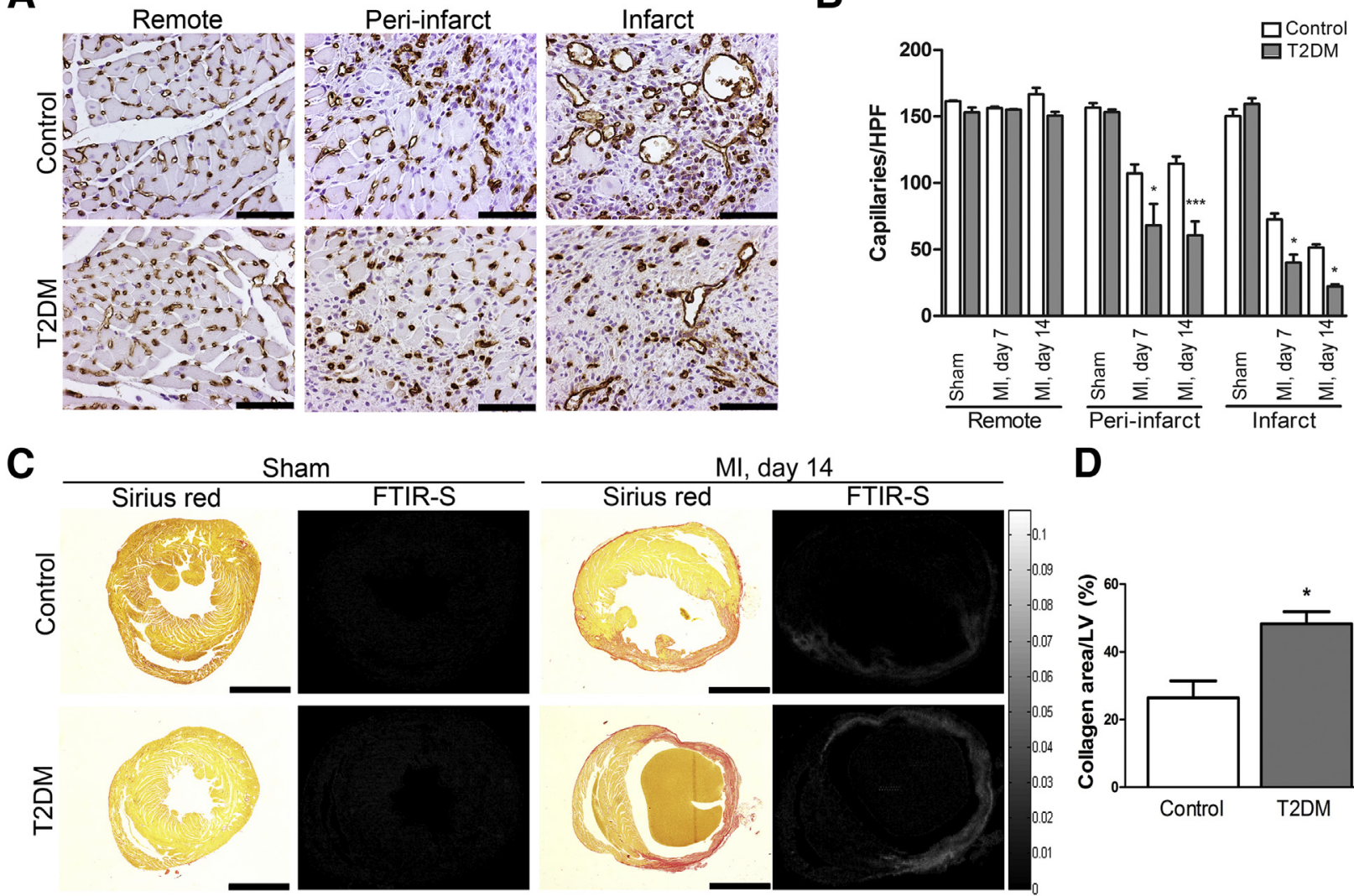

D

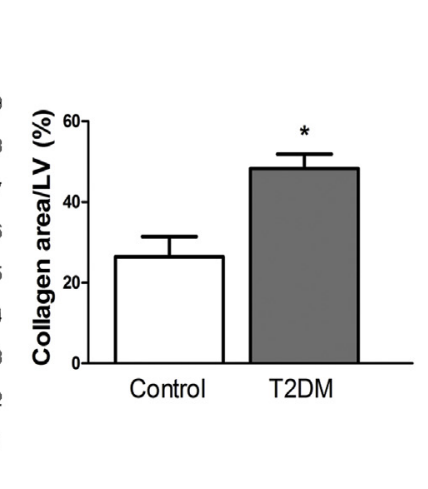

Figure 6 Impaired neovascularization and increased infarct and fibrotic area after myocardial infarction (MI) in type 2 diabetes mellitus (T2DM). A: Representative images showing capillary endothelial staining with CD31 in peri-infarct and infarct regions of ischemic left ventricle (LV) at day 7 after MI. B: Quantification of $\mathrm{CD}_{3} 1^{+}$capillaries from remote, peri-infarct, and infarct regions of LV of sham and infarcted hearts at day 7 and 14 after MI. C: Representative images from Sirius Red staining for collagen deposition in the ischemic LV together with representative images from Fourier transform infrared spectroscopy (FTIR-S) mapping for collagen-I. D: Quantification of collagen-positive area from whole LV at day 14 after MI. Data are expressed as means \pm SEM (B and D). $n=4$ to $5 .{ }^{*} P<0.05$ T2DM versus controls, one-way analysis of variance with Bonferroni's multiple comparison test. Scale bars: $50 \mu \mathrm{m}(\mathbf{A}) ; 2000 \mu \mathrm{m}(\mathbf{C})$. HPF, high power field.

were up-regulated and 1041 were down-regulated as shown in the scatter plots in Figure 2A, volcano plots in Figure 2B and Supplemental Table $\mathrm{S} 1,{ }^{20-22}$ and Supplemental Table S2. Ingenuity functional annotation suggested an increased cell death, necrosis, movement disorders, heart disease, neuromuscular disease, hypertrophy, and cardiomyopathy, and decreased metabolism of nucleic acid components, metabolism of nucleotides, heart rate, and contractility of muscles in T2DM mice compared with controls as shown in Table 2, Supplemental Table $\mathrm{S} 1,{ }^{20-22}$ and Supplemental Table S3. Ingenuity pathways analysis revealed mitochondrial dysfunction, tricarboxylic acid (TCA) cycle, and fatty acid $\beta$-oxidation as the most prominent pathways affected by T2DM in the LV after MI as shown in Figure 2C and Supplemental Table S4. Ingenuity upstream regulator analysis suggested an inhibition of peroxisome proliferator-activated receptor $\gamma$ coactivator (PGC)-1 $\alpha$ regulatory network (essential regulator of cardiac metabolism and mitochondrial homeostasis) consisting of coregulators PGC- $1 \alpha$ and PGC$1 \beta$; nuclear receptors ESRRA, ESRRG, PPARA, PPARG,
PPARD; other transcription factors TFAM, HAND2, GATA4; and activation of STAT1, NR3C2, NRIP1, SMAD3, IRF3, NFATC2, SPI1 in T2DM mice compared with controls as shown in Table 3 and Supplemental Table S5. Collectively, these data reveal that T2DM dysregulates genes important in cardiac function, mitochondrial homeostasis, cell death, and metabolism of nucleic acids in LV after MI.

\section{Impaired Mitophagy in Cardiomyocytes of Peri-Infarct Region in T2DM Hearts}

Genes critical in mitochondrial fusion machinery and regeneration of healthy mitochondria, including $M f n l$, Mfn2, Opal, and genes involved in removal of damaged mitochondria and autophagy, including, Lc3a and Pinkl, were significantly down-regulated in peri-infarct regions of LV of T2DM mice compared with controls at day 7 after MI (Figure 3A, Supplemental Table S1, ${ }^{20-22}$ and Supplemental Table S2). To unravel cell specificity of differential gene expression, we isolated cardiomyocytes from peri-infarct 
and infarct regions using Langendorff perfusion procedure. Subsequently, noncardiomyocyte fractions were used for cardiac macrophage isolation by using a combination of magnetic-assisted cell sorting with FACS. ${ }^{14}$ Cell-specific gene expression showed decreased activity of mitochondrial fusion and autophagy-related genes predominantly in cardiomyocytes from peri-infarct regions of LV of T2DM compared with controls (Figure 3B). In addition, Western blot analysis showed increased LC3-II/I ratio and p62 levels in left ventricular homogenates from T2DM mice compared with controls, indicating impaired mitophagy/autophagy (Figure 3C). Electron microscopic images showed an ordered array of myofibers arranged with mitochondria stacked in between them in cardiomyocytes of control mice. In addition, intramyocellular lipid deposition was seen in between mitochondria in the T2DM mice at baseline, similar to observations in diabetic human hearts. Mitophagy was activated in the peri-infarct regions of LV of both groups at day 7 after MI. Of interest, cardiomyocytes in peri-infarct regions of $\mathrm{LV}$ in T2DM showed increased accumulation of undegraded mitochondria in autophagosomes (Figure 3D). Immunoelectron microscopic analysis with anti-DNA antibody showed localization in autolysosomes, suggesting the release of undegraded mtDNA into cytosol as a consequence of impaired autolysosomal degradation (Figure 3E). Interestingly, our RNA-sequencing analysis suggested decreased metabolism of nucleic acids and nucleotides in LV of T2DM mice, corroborating with impaired mitophagy. In addition, immunofluorescence staining showed increased accumulation of LC3-positive mitophagosomes/autophagosomes, together with increased p62 levels in peri-infarct regions of $\mathrm{LV}$ in T2DM mice compared with controls, further suggesting impaired mitophagy/autophagy as a potential mechanism of release of mitochondrial-derived DAMPs intracellularly and extracellularly after cell death (Figure 3F).

\section{Exaggerated Inflammasome Activation and Caspase-I Activity in T2DM Hearts}

Genes involved in cytosolic dsDNA sensing (Ifi204, pyhin1), inflammasome (Aim2, NLRC4) and caspase-I were significantly up-regulated in peri-infarct and infarct regions of LV in T2DM mice compared with controls at day 7 after MI (Figure 4A, Supplemental Table S1, ${ }^{20-22}$ Supplemental Table S2). To dissect out the cell-specific roles of inflammasome in cardiomyocytes and macrophages, these cell populations were isolated, and Pyhin1, Ifi204, Aim2, NLRC4, Eif2ak2, and Caspase-1 were looked at for gene expression. Of note, all these genes were significantly upregulated both in cardiomyocytes (except Eif2ak2) and macrophages isolated from T2DM mice compared with controls (Figure 4B). Furthermore, immunofluorescence double stainings for cardiomyocyte- and macrophagespecific dsDNA sensors (Pyhinl, Ifi204) showed increased activation both in cardiomyocytes and macrophages from peri-infarct and infarct regions of LV in T2DM compared with controls at day 7 after MI (Supplemental Figure S1). Similarly, immunofluorescence double stainings for cardiomyocyte- and macrophagespecific inflammasome (Aim2, NLRC4) showed abundance of double-positive cells in peri-infarct and infarct regions of LV in T2DM compared with controls at day 7 after MI (Figure 4, C and D).

Inflammasome activation culminates in either caspaseI-mediated cell death (pyroptosis) or cytokine processing into mature active forms (IL-1 $\beta$, IL-18). To understand the consequence of inflammasome activation in this context, caspase-I activity was examined by immunostainings of left ventricular tissue collected at day 7 after MI. Similar to Aim2 and NLRC4 stainings, caspase-1 immunostaining showed increased activation in peri-infarct and infarct regions of LV in both groups (Figure 4E). In addition, caspase-1 colorimetric assay using left ventricular tissue homogenates showed increased activity in T2DM mice compared with controls (Figure 4G). Next, cell death was examined with TUNEL assay, similar to caspase-I stainings an increased number of TUNEL-positive cells were observed in peri-infarct and infarct regions of $\mathrm{LV}$ of T2DM mice compared with controls (Figure 4, F and H). In parallel with increased inflammasome and caspase-I activity, increased caspase-1-dependent inflammatory mediator (IL-18) was observed in circulation of T2DM mice compared with controls at baseline and day 7 after MI (Figure 4I). Of note, increased inflammation as evidenced by increased IL-18, TNF- $\alpha$, and circulating immune cells were shown to associate with remodeling and HF after MI in patients. ${ }^{23,24}$ Collectively, these results indicated that exaggerated activation of Aim2 and NLRC4 inflammasome and subsequent increase in caspase-I-mediated pathways in cardiomyocytes and macrophages in periinfarct and infarct regions of LV of T2DM mice compared with controls.

\section{Altered Cardiac Macrophage Phenotypic Responses in T2DM Hearts}

Double immunofluorescence staining of left ventricular tissue for M2-like macrophage (anti-F4/80 and antiArginase-1) showed decreased numbers of M2-like macrophages in peri-infarct and infarct regions in LV of T2DM mice compared with controls. On the contrary, stainings for M1-like macrophage (anti-F4/80 and anti-TNF- $\alpha$ ) showed an increase in T2DM mice compared with controls at day 7 after MI (Figure 5A). Furthermore, flow cytometric analysis was performed for M2-like macrophages in left ventricular tissues collected at day 7 after MI as described previously. ${ }^{14} \mathrm{CD} 45^{+} \mathrm{F} 4 / 80^{+} \mathrm{CD} 206^{+}$M2-like macrophages were analyzed from sham and infarcted hearts of control and T2DM mice (Figure 5B). Quantification suggested a significant decline in M2-like macrophages in LV of T2DM mice compared with controls 
(Figure 5C). Collectively, these results indicated an altered macrophage phenotypic response to $\mathrm{MI}$ in $\mathrm{LV}$ of T2DM mice, consistent with exaggerated inflammasome activation.

\section{Impaired Neovascularization and Increased Fibrosis in T2DM Hearts}

Neovascularization responses after MI were examined with capillary endothelial staining with $\mathrm{CD} 31\left(\mathrm{CD} 31^{+}\right.$cells). Quantification of $\mathrm{CD} 31^{+}$cells showed a significantly decreased number of capillaries in peri-infarct and infarct regions of LV in T2DM mice compared with controls at day 7 and 14 after MI (Figure 6, A and B). Corroborating with altered macrophage phenotype, capillary stainings demonstrated an impaired neovascularization after MI in LV of T2DM mice compared with controls.

Infarct size and myocardial fibrosis were evaluated with Sirius Red staining and Fourier transform infrared spectroscopic mapping of collagen-I at baseline and day 14 after MI (Figure 6C). Interestingly, Sirius Red staining and Collagen-I mapping by Fourier transform infrared spectroscopy showed similar patterns of collagen distribution in LVs of both control and T2DM mice. Quantification showed significantly increased area of infarction and collagen-I deposition, especially in LV of T2DM mice compared with controls (Figure 6D). Increased fibrosis has been suggested to disrupt the excitation-contraction coupling between cardiomyocytes, and a subsequent increase in stiffness will lead to decreased ventricular wall compliance, resulting in both systolic and diastolic dysfunction. $^{25}$ Thus, impaired neovascularization responses coupled with increased fibrosis after MI might further decrease cardiac contractility and left ventricular $\mathrm{EF}$ of the heart after MI in T2DM mice compared with controls.

\section{Discussion}

In our previous study, we characterized a mouse model of insulin resistance and T2DM, which demonstrates typical features of human T2DM, including insulin resistance, hyperglycemia, and mild hyperinsulinemia. ${ }^{13}$ In addition, these mice show an increased atherosclerotic progression and lesion calcification. Recently, with the use of these T2DM mice we have demonstrated an impaired vascular growth in response to hindlimb ischemia as a consequence of altered macrophage phenotype. ${ }^{14}$ In the present study, we provide insights into pathways underlying accelerated $\mathrm{HF}$ after MI in T2DM. Transcriptional profiling revealed an increased cell death, necrosis, myocardial dysfunction and decreased heart rate, cardiac contractility, and metabolism of nucleic acids. Integrating transcriptomics and molecular characterization, we show an impaired mitophagy associated with increased accumulation of undegraded mitochondria in autophagosomes in cardiomyocytes from peri-infarct regions of the LV. Furthermore, our immunogold labeling studies suggested localization of mtDNA to autolysosomes in cardiomyocytes of LV, indicating increased release of undegraded mtDNA into cytosol as a result of impaired autolysosomal degradation of dysfunctional mitochondria. Reflecting increased cytosolic mtDNA as a result of impaired mitophagy, cytoplasmic DNA sensors, including Pyhin1, Ifi204, and inflammasome, namely AIM2, NLRC4, and Caspase-I, were highly activated in cardiomyocytes and macrophages in peri-infarct and infarct regions of the LV. This was associated with increased cell death and IL-18 secretion, thus linking defective mitophagy to inflammasome activation (Supplemental Figure S2). Moreover, cardiac macrophages showed an altered phenotype consistent with inflammasome activation in LV of T2DM mice.

Mitophagy is a selective form of autophagy involved in the removal of damaged mitochondria during cellular stress, which prevents the release of mitochondria-derived DAMPS to the cytoplasm. In addition, mitophagy prevents activation of prodeath pathways in favor of adaptation to stress. ${ }^{26} \mathrm{We}$ observed an increased number of autophagosomes that contained undegraded mitochondria (mitophagy) but reduced amounts of autolysosomes in cardiomyocytes from peri-infarct regions of LV in T2DM mice together with an increased LC3-II-to-LC3-I ratio and p62 levels. Furthermore, immunofluorescence staining showed an increased accumulation of LC3-positive mitophagosomes/autophagosomes, together with increased p62 levels, further suggesting impaired mitophagy. Given the abundance of mitochondria in cardiomyocytes, it is likely that a substantial amount of mitochondrial DNA would be released extracellularly in the heart on tissue damage as a result of impaired mitophagy. ${ }^{27}$ Consistent with this notion, immunoelectron microscopic analysis with antiDNA antibody showed localization in autolysosomes. In addition, our RNA sequencing analysis suggested decreased metabolism of nucleic acids and nucleotides in LV of T2DM, supporting the notion that impaired degradation of mtDNA as a result of insufficient mitophagy might lead to increased release of undegraded mtDNA. Thus, impaired mitophagy-associated DAMPs likely contribute to exaggerated inflammasome activation and cell death. Our observations are in line with a recent study that found increased inflammation and cardiac dysfunction in response to transaortic constriction, as a result of mtDNA release from inefficient degradation by autophagy. ${ }^{28}$

Inflammasome is a multiprotein complex that activates Caspase-1, triggering an inflammatory cell death called pyroptosis. In addition, caspase- 1 triggers the maturation and release of proinflammatory cytokines IL- $1 \beta$ and IL$18{ }^{29}$ Indeed, inflammasome activation in the infiltrated inflammatory cells are shown to enhance myocardial ischemia-reperfusion injury. Of interest, almost all cardiac 
macrophages expressed inflammasome adaptor apoptosisassociated speck-like protein containing C-terminal caspase recruitment domain (ASC), whereas approximately $60 \%$ of neutrophils expressed ASC. The expression of caspase-1 was visualized mainly in the infiltrated cells of the ischemic myocardium. ${ }^{30}$ NLRP3 inflammasome activation in cardiomyocytes and cardiac leukocytes in response to ATP released from dying cells was shown to play an important role in adverse cardiac remodeling in response to MI under nondiabetic conditions. ${ }^{31}$ Thus, inflammasome-dependent caspase-I activation and subsequent release of IL-18 and IL-1 $\beta$, both in cardiomyocytes and macrophages, seems to lead to HF. Accordingly, inhibition of caspase-I reduced cardiomyocyte death, improved cardiac function, and significantly decreased rates of mortality. ${ }^{32-34}$ Transgenic mice overexpressing caspase-1 show an increased cardiomyocyte cell death and HF after MI, independent of increase in IL- $1 \beta$ and IL-18 secretion. ${ }^{35}$ Collectively, our findings suggest that defective mitophagy in T2DM contributes to $\mathrm{HF}$ after MI through exaggerated inflammasome activation, subsequent cell death, and cytokine secretion.

Our results suggest an increased inflammasome activation in cardiac macrophages from T2DM mice compared with control mice. In parallel, immunofluorescence stainings and flow cytometric analysis suggested a decrease in reparative M2-like macrophages but an increase in inflammatory M1like macrophages in LV of T2DM mice. Consistent with altered macrophage phenotype, T2DM displayed impaired neovascularization and increased fibrosis in peri-infarct and infarct regions in the LV at day 14 after MI. Although both M1- and M2-macrophages are critical for resolving myocardial injury, it has become increasingly evident that shifting the balance of macrophage to an M2-like phenotype is cardioprotective. ${ }^{36}$ On the contrary, the delayed phenotypic transition of M1-like macrophages to M2-like macrophages and/or prolonged and increased recruitment of inflammatory monocytes to the heart leads to impaired infarct healing and increased $\mathrm{HF}$ after MI as demonstrated in atherosclerotic mice. ${ }^{37,38}$ In line with these observations, we found skewing of cardiac macrophages toward a proinflammatory phenotype in T2DM hearts, associated with impaired neovascularization and increased fibrosis compared with reparative macrophage phenotype in control hearts.

We recognize that our study has limitations. Comparisons were made between T2DM mice with $\mathrm{C} 57 \mathrm{BL} / \mathrm{J}$ controls to keep all mice on the same genetic background which can have significant effects on the measured variables. In addition, aged mice with high-fat diet were used to aggravate the T2DM phenotype. ${ }^{13}$ However, we cannot exclude a possibility that other factors such as age, insulin resistance, or high-fat diet could have contributed to the observed differences in response to MI. Despite these limitations, our findings suggest that T2DM significantly contributed to aggravated post-infarct $\mathrm{HF}$.

\section{Conclusions}

Our results suggest that an impaired mitophagy and exaggerated inflammasome activation together with altered macrophage phenotypic responses may contribute to HF after MI in T2DM. Modulation of mitophagy together with inhibition of inflammasome activation may offer a novel therapeutic target.

\section{Acknowledgments}

We thank Svetlana Laidinen for help in LAD operations and The Sequencing Service GeneCore Sequencing Facility (EMBL Heidelberg, Germany) for mRNA sequencing service.

\section{Supplemental Data}

Supplemental material for this article can be found at https://doi.org/10.1016/j.ajpath.2017.08.023.

\section{References}

1. Grundy SM, Benjamin IJ, Burke GL, Chait A, Eckel RH, Howard BV Mitch W, Smith SC Jr, Sowers JR: Diabetes and cardiovascular disease: a statement for healthcare professionals from the American Heart Association. Circulation 1999, 100:1134-1146

2. From AM, Leibson CL, Bursi F, Redfield MM, Weston SA, Jacobsen SJ, Rodeheffer RJ, Roger VL: Diabetes in heart failure: prevalence and impact on outcome in the population. Am J Med 2006, 119:591-599

3. Bornfeldt KE, Tabas I: Insulin resistance, hyperglycemia, and atherosclerosis. Cell Metab 2011, 14:575-585

4. Haffner SM, Lehto S, Ronnemaa T, Pyorala K, Laakso M: Mortality from coronary heart disease in subjects with type 2 diabetes and in nondiabetic subjects with and without prior myocardial infarction. $\mathrm{N}$ Engl J Med 1998, 339:229-234

5. Zuanetti G, Latini R, Maggioni AP, Santoro L, Franzosi MG: Influence of diabetes on mortality in acute myocardial infarction: data from the GISSI-2 study. J Am Coll Cardiol 1993, 22:1788-1794

6. Sena S, Hu P, Zhang D, Wang X, Wayment B, Olsen C, Avelar E, Abel ED, Litwin SE: Impaired insulin signaling accelerates cardiac mitochondrial dysfunction after myocardial infarction. J Mol Cell Cardiol 2009, 46:910-918

7. Go AS, Mozaffarian D, Roger VL, Benjamin EJ, Berry JD, Borden WB, et al; American Heart Association Statistics Committee and Stroke Statistics Subcommittee: Heart disease and stroke statistics-2013 update: a report from the American Heart Association. Circulation 2013, 127:e6-e245

8. Cubbon RM, Wheatcroft SB, Grant PJ, Gale CP, Barth JH, Sapsford RJ, Ajjan R, Kearney MT, Hall AS; Evaluation of Methods and Management of Acute Coronary Events Investigators: Temporal trends in mortality of patients with diabetes mellitus suffering acute myocardial infarction: a comparison of over 3000 patients between 1995 and 2003. Eur Heart J 2007, 28:540-545

9. Norhammar A, Lindback J, Ryden L, Wallentin L, Stenestrand U; Register of Information and Knowledge about Swedish Heart Intensive Care Admission (RIKS-HIA): Improved but still high short- and longterm mortality rates after myocardial infarction in patients with diabetes mellitus: a time-trend report from the Swedish Register of 
Information and Knowledge about Swedish Heart Intensive Care Admission. Heart 2007, 93:1577-1583

10. Frangogiannis NG: The immune system and cardiac repair. Pharmacol Res 2008, 58:88-111

11. Liehn EA, Postea O, Curaj A, Marx N: Repair after myocardial infarction, between fantasy and reality: the role of chemokines. J Am Coll Cardiol 2011, 58:2357-2362

12. van der Laan AM, Nahrendorf M, Piek JJ: Healing and adverse remodelling after acute myocardial infarction: role of the cellular immune response. Heart 2012, 98:1384-1390

13. Heinonen SE, Leppanen P, Kholova I, Lumivuori H, Hakkinen SK, Bosch F, Laakso M, Yla-Herttuala S: Increased atherosclerotic lesion calcification in a novel mouse model combining insulin resistance, hyperglycemia, and hypercholesterolemia. Circ Res 2007, 101: 1058-1067

14. Babu M, Durga Devi T, Makinen P, Kaikkonen M, Lesch HP, Junttila S, Laiho A, Ghimire B, Gyenesei A, Yla-Herttuala S: Differential promoter methylation of macrophage genes is associated with impaired vascular growth in ischemic muscles of hyperlipidemic and type 2 diabetic mice: Genome-Wide Promoter Methylation Study. Circ Res 2015, 117:289-299

15. Gao E, Lei YH, Shang X, Huang ZM, Zuo L, Boucher M, Fan Q, Chuprun JK, Ma XL, Koch WJ: A novel and efficient model of coronary artery ligation and myocardial infarction in the mouse. Circ Res 2010, 107:1445-1453

16. Ingolia NT, Ghaemmaghami S, Newman JR, Weissman JS: Genomewide analysis in vivo of translation with nucleotide resolution using ribosome profiling. Science 2009, 324:218-223

17. Cheheltani R, Rosano JM, Wang B, Sabri AK, Pleshko N, Kiani MF: Fourier transform infrared spectroscopic imaging of cardiac tissue to detect collagen deposition after myocardial infarction. J Biomed Opt 2012, 17:056014

18. Mizushima N, Yoshimori T, Levine B: Methods in mammalian autophagy research. Cell 2010, 140:313-326

19. Mutikainen M, Tuomainen T, Naumenko N, Huusko J, Smirin B, Laidinen S, Kokki K, Hynynen H, Yla-Herttuala S, Heinaniemi M, Ruas JL, Tavi P: PGC-1alpha1 induces cardiac excitation-contraction coupling phenotype without metabolic remodelling. J Physiol 2016, 594:7049-7071

20. Ingersoll MA, Spanbroek R, Lottaz C, Gautier EL, Frankenberger M, Hoffmann R, Lang R, Haniffa M, Collin M, Tacke F, Habenicht AJ, Ziegler-Heitbrock L, Randolph GJ: Comparison of gene expression profiles between human and mouse monocyte subsets. Blood 2010, 115:e10-e19

21. Kadl A, Meher AK, Sharma PR, Lee MY, Doran AC, Johnstone SR, Elliott MR, Gruber F, Han J, Chen W, Kensler T, Ravichandran KS, Isakson BE, Wamhoff BR, Leitinger N: Identification of a novel macrophage phenotype that develops in response to atherogenic phospholipids via Nrf2. Circ Res 2010, 107:737-746

22. Ostuni R, Piccolo V, Barozzi I, Polletti S, Termanini A, Bonifacio S, Curina A, Prosperini E, Ghisletti S, Natoli G: Latent enhancers activated by stimulation in differentiated cells. Cell 2013, 152:157-171

23. Johansson A, Eriksson N, Becker RC, Storey RF, Himmelmann A, Hagstrom E, Varenhorst C, Axelsson T, Barratt BJ, James SK, Katus HA, Steg PG, Syvanen AC, Wallentin L, Siegbahn A; PLATO Investigators: NLRC4 inflammasome is an important regulator of interleukin-18 levels in patients with acute coronary syndromes: Genome-Wide Association Study in the PLATelet inhibition and patient Outcomes Trial (PLATO). Circ Cardiovasc Genet 2015, 8: 498-506

24. Engstrom G, Melander O, Hedblad B: Leukocyte count and incidence of hospitalizations due to heart failure. Circ Heart Fail 2009, 2: 217-222

25. Asbun J, Villarreal FJ: The pathogenesis of myocardial fibrosis in the setting of diabetic cardiomyopathy. J Am Coll Cardiol 2006, 47: 693-700

26. Kubli DA, Gustafsson AB: Mitochondria and mitophagy: the yin and yang of cell death control. Circ Res 2012, 111:1208-1221

27. Shimada K, Crother TR, Karlin J, Dagvadorj J, Chiba N, Chen S, Ramanujan VK, Wolf AJ, Vergnes L, Ojcius DM, Rentsendorj A, Vargas M, Guerrero C, Wang Y, Fitzgerald KA, Underhill DM, Town T, Arditi M: Oxidized mitochondrial DNA activates the NLRP3 inflammasome during apoptosis. Immunity 2012, 36:401-414

28. Oka T, Hikoso S, Yamaguchi O, Taneike M, Takeda T, Tamai T, Oyabu J, Murakawa T, Nakayama H, Nishida K, Akira S, Yamamoto A, Komuro I, Otsu K: Mitochondrial DNA that escapes from autophagy causes inflammation and heart failure. Nature 2012, 485:251-255

29. Bergsbaken T, Fink SL, Cookson BT: Pyroptosis: host cell death and inflammation. Nat Rev Microbiol 2009, 7:99-109

30. Kawaguchi M, Takahashi M, Hata T, Kashima Y, Usui F, Morimoto H, Izawa A, Takahashi Y, Masumoto J, Koyama J, Hongo M, Noda T, Nakayama J, Sagara J, Taniguchi S, Ikeda U: Inflammasome activation of cardiac fibroblasts is essential for myocardial ischemia/reperfusion injury. Circulation 2011, 123: 594-604

31. Mezzaroma E, Toldo S, Farkas D, Seropian IM, Van Tassell BW, Salloum FN, Kannan HR, Menna AC, Voelkel NF, Abbate A: The inflammasome promotes adverse cardiac remodeling following acute myocardial infarction in the mouse. Proc Natl Acad Sci U S A 2011, 108:19725-19730

32. Pomerantz BJ, Reznikov LL, Harken AH, Dinarello CA: Inhibition of caspase 1 reduces human myocardial ischemic dysfunction via inhibition of IL-18 and IL-1beta. Proc Natl Acad Sci U S A 2001, 98: 2871-2876

33. Frantz S, Ducharme A, Sawyer D, Rohde LE, Kobzik L, Fukazawa R, Tracey D, Allen H, Lee RT, Kelly RA: Targeted deletion of caspase-1 reduces early mortality and left ventricular dilatation following myocardial infarction. J Mol Cell Cardiol 2003, 35:685-694

34. Holly TA, Drincic A, Byun Y, Nakamura S, Harris K, Klocke FJ, Cryns VL: Caspase inhibition reduces myocyte cell death induced by myocardial ischemia and reperfusion in vivo. J Mol Cell Cardiol 1999, 31:1709-1715

35. Merkle S, Frantz S, Schon MP, Bauersachs J, Buitrago M, Frost RJ, Schmitteckert EM, Lohse MJ, Engelhardt S: A role for caspase-1 in heart failure. Circ Res 2007, 100:645-653

36. Frangogiannis NG: Regulation of the inflammatory response in cardiac repair. Circ Res 2012, 110:159-173

37. Nahrendorf M, Pittet MJ, Swirski FK: Monocytes: protagonists of infarct inflammation and repair after myocardial infarction. Circulation 2010, 121:2437-2445

38. Panizzi P, Swirski FK, Figueiredo JL, Waterman P, Sosnovik DE, Aikawa E, Libby P, Pittet M, Weissleder R, Nahrendorf M: Impaired infarct healing in atherosclerotic mice with Ly-6C(hi) monocytosis. J Am Coll Cardiol 2010, 55:1629-1638 